Archives

$24 \mid 2000$

La séduction

\title{
L'arbitrisme : un concept d'historien ?
}

\section{Anne Dubet}

\section{(2) OpenEdition}

\section{Journals}

Édition électronique

URL : http://journals.openedition.org/ccrh/2062

DOI : $10.4000 /$ ccrh.2062

ISSN : 1760-7906

Éditeur

Centre de recherches historiques - EHESS

Édition imprimée

Date de publication : 20 avril 2000

ISSN : 0990-9141

Référence électronique

Anne Dubet, "L'arbitrisme : un concept d'historien? ", Les Cahiers du Centre de Recherches Historiques [En ligne], 24 | 2000, mis en ligne le 17 janvier 2009, consulté le 19 avril 2019. URL : http:// journals.openedition.org/ccrh/2062 ; DOI : 10.4000/ccrh.2062

Ce document a été généré automatiquement le 19 avril 2019

Article L.111-1 du Code de la propriété intellectuelle. 


\title{
L'arbitrisme : un concept d'historien?
}

\author{
Anne Dubet
}

\section{NOTE DE L'AUTEUR}

Abreviations : leg. : legajo(s) : ACC : Actas de las Cortes de Castilla y Léon ; AA.CC. : Actas Capitulares; MCV : Mélanges de la Casa Velázquez; AGS : Archives Générales deSimancas ; AVM : Archives de la ville de Madrid ; BNM : Bibliothèque Nationale (Madrid).

Longtemps oubliés ou méprisés par les historiens, ceux que l'on appelle communément les arbitristes (arbitristas) suscitent depuis quelques lustres l'intérêt des chercheurs. Ils trouvent chez eux soit un témoignage et une réflexion critique sur la « décadence », soit son revers, à savoir l'expression d'une volonté réformatrice. Deux études importantes, le livre consacré par Jean Vilar à la satire de l'arbitriste ${ }^{1}$ et la synthèse plus récente de JuanIgnacio Gutiérrez Nieto ${ }^{2}$, sont devenues des références ${ }^{3}$. Parallèlement, depuis le milieu des années 1970, les mots arbitriste et arbitrisme, absents des dictionnaires courants du français, sont entrés dans le vocabulaire des historiens francophones ${ }^{4}$. La plupart des études de la pratique politique ou des conceptions économiques et politiques des sujets $\mathrm{du}$ Roi Catholique aux $\mathrm{XVI}^{\mathrm{e}}$ et $\mathrm{XVII}^{\mathrm{e}}$ siècles abordent l'arbitrisme. Il ne paraît donc plus nécessaire de revenir sur le sens de ces mots. Mon intention n'est pas de reprendre ici sur toute l'historiographie des arbitristes ${ }^{5}$. C'est sur le phénomène dénommé arbitrisme qu'il convient de s'interroger. En effet, contrairement à arbitrista, que l'on trouve dans la littérature espagnole depuis le début du XVII ${ }^{\mathrm{e}}$ siècle et que les dictionnaires ont accueilli dès le XVIII ${ }^{\mathrm{e}}$, le mot arbitrismo et son équivalent français paraissent être une création récente des historiens ${ }^{7}$. Or, si ce terme présente l'avantage de nous éviter le détour d'une périphrase - et il sera employé à l'envi dans cet article -, on peut se demander s'il ne crée pas une «illusion de réalité ». Alors que les arbitristes n'ont jamais fait l'objet de l'étude sociologique réclamée par John $\mathrm{H}$. Elliot ${ }^{8}$, on passe aisément de l'idée que l'arbitrisme constitue un objet historique digne d'intérêt au postulat implicite qu'il s'agit d'une réalité discernable et repérable. Autrement dit, il y aurait une homogénéité entre les arbitristes, 
crédités d'une "pensée économique, politique et sociale » unificatrice. On les traite ainsi comme des êtres à part dans la société d'Ancien Régime, en faisant l'économie d'une réflexion sur le fonctionnement de l'administration royale et sur ses relations avec les autres centres de pouvoir.

On tentera donc de répondre à quelques questions : peut-on identifier le groupe arbitriste et en tracer les limites? Y a-t-il un arbitrisme en tant que mouvement ou des arbitristes? Quelle est leur place dans le système politique d'Ancien Régime? Comment s'y prendre pour étudier un objet dont la réalité apparaît problématique?

\section{À l'origine, la satire}

3 Point n'est besoin, après les travaux de Jean Vilar, de revenir longuement sur la naissance du stéréotype littéraire de l'arbitriste et de son pendant «réel». En Espagne, les premières occurrences du terme arbitrista apparaissent au début $\mathrm{du}$ XVII ${ }^{\mathrm{e}}$ siècle dans la littérature, tandis que dès 1588 les délégués des villes (procuradores) aux Cortes de Castille et Léon dénoncent les auteurs d'arbitrios 9 .

4 L'arbitriste en Espagne, à l'instar de son équivalent en France, le donneur d'avis ${ }^{10}$, est un auteur de mémoires qui s'adresse au roi, à ses Conseils, à ses juntes (juntas) ${ }^{11}$,ou à quelque membre influent de ces organismes, aux Cortes ou aux parlements, afin de leur indiquer les mesures à prendre pour sortir de difficultés d'ordre financier, fiscal ou économique. Ces offres ne se font pas sans contrepartie : plusieurs historiens ont décrit la procédure par laquelle l'arbitriste remet d'abord une brève description de son arbitrio; si celui-ci est original, c'est-à-dire s'il ne figure pas dans les livres de l'organisme sollicité, ce dernier émettra au nom du roi un privilège : celui-ci garantit à l'arbitriste un "droit d'avis » (un pourcentage des bénéfices de l'ordre de $4 \%$ au XVII ${ }^{e}$ siècle) si son invention est mise à exécution ${ }^{12}$. Dans d'autres cas, la demande de gain est moins explicite, quoique l'arbitriste attende toujours d'être récompensé pour le service rendu.

Le large éventail des mesures imaginées a été décrit. On y inclut des expédients à court terme, dont l'application, limitée dans le temps et dans l'espace, doit assurer à la Couronne des bénéfices immédiats : par exemple, une vente d'office, la levée d'une taxe sur les aliments ou le contrôle des comptes d'un trésorier ou d'un receveur des impôts indélicat, dont on peut prouver qu'il doit de l'argent au roi. Mais l'histoire a qualifié d'arbitristes les tenants de politiques plus ambitieuses, qui impliquent une réforme de l'administration fiscale et une transformation des pratiques financières, voire de l'organisation de la société et de ses valeurs. Parmi les plus célèbres : Cristóbal Pérez de Herrera, Sancho de Moncada, Martin González de Cellorigo ou encore Luis Valle de la Cerda $^{13}$.

6 Jean Vilar a décelé dans la satire des discours écrits par les arbitristes et dans les arbitrios réels qu'il a consultés certaines constantes, d'ordre formel ou thématique. Au cœur de leurs préoccupations, l'extinction de la dette consolidée du roi et le désengagement ( desempeño) de son patrimoine, la réduction de la pression fiscale - accusée de décourager les artisans, les paysans et les marchands et de pousser les plus pauvres à l'exode rural ou à l'exil, tandis que les plus fortunés se réfugient dans l'oisiveté rentière -, la reprise de l'agriculture ou encore la lutte contre les effets pervers de l'inflation de billon. C'est une rhétorique commune, qui oppose les dommages aux remèdes, les maux universels au bien commun. Ainsi, nombre d'arbitristes se disent porteurs des meilleures solutions ou du 
remède unique qui arrachera le corps de la monarchie à la maladie et au déclin ( declination), et qui assurera au contraire sa conservation, son accroissement ou sa restauration (conservación, aumento, restauración). Le remède, dans tous les cas, est facile, peu coûteux pour le roi, et souvent susceptible de lui procurer des avantages politiques indéniables (l'amour de ses sujets). La satire insiste sur son caractère contradictoire : on raille les arbitristes qui proposent de réduire la pression fiscale tout en élevant les recettes $\mathrm{du}$ roi. Parfois, les arbitrios sont tout bonnement absurdes: l'arbitriste de Cervantès oblige tous les sujets adultes à jeûner une fois par mois et à remettre au roi le prix de leur repas ${ }^{14}$.

7 Corollaire : les arbitristes - tout comme en France les donneurs d'avis - ne peuvent être vus de façon mécanique. On leur prête des caractéristiques psychologiques et morales. Les écrivains soucieux de ne pas être confondus avec les arbitristes, les dramaturges et les romanciers, leur attribuent des traits psychologiques peu enviables : ils sont orgueilleux (ils détiennent la panacée), âpres au gain (le remède se vend), peu soucieux du bien-être du peuple (ils l'accableront d'impôts). Leurs idées peuvent même être l'expression d'une folie caractérisée (on les trouvera dans des asiles). Aux yeux des gens de cour auxquels ils remettent leurs projets, ce sont aussi des «fâcheux » - si l'on en croit Molière - dont on a le plus grand mal à se défaire sans bourse délier.

\section{Des obstacles à l'appréhension d'un concept}

8 Ce portrait de l'arbitriste, qui tient lieu de définition, pose un certain nombre de problèmes au moment d'aborder le phénomène de l'«arbitrisme ", et ceci pour trois raisons : 1) l'arbitriste est méprisable, 2) le portrait qu'on en fait est essentiellement psychologique, non sociologique, ce qui contribue à l'éloigner du reste de la société, 3) enfin, on confond parfois les sujets de prédilection des arbitristes (notamment la crise économique) avec les raisons de leur existence.

\section{Le mépris de l'arbitriste}

9 Le portrait qui vient d'être rapidement brossé de l'arbitriste tel que la satire littéraire le représente a longtemps trouvé écho chez les historiens. On connaît l'opinion de Manuel Colmeiro, qui reprochait aux arbitristes leur orgueil et l'extravagance de leurs projets, responsables de la décadence de l'Espagne ${ }^{15}$. Des historiens plus récents tiennent les arbitrios pour des élucubrations dangereuses pour le contribuable ${ }^{16}$ ou bien s'en prennent à leurs erreurs et à leur ignorance des vérités révélées de l'économie libérale ${ }^{17}$.

Cette attitude paraît révolue depuis qu'Earl J. Hamilton, Pierre Vilar, José Larraz, John H. Elliott et Jean Vilar nous ont invités à considérer d'un autre oil la production des arbitristes $^{18}$. Les écrits des "primitifs espagnols ", théologiens de l'école de Salamanque ou écrivains comme Luis de Ortiz - souvent considéré comme l'initiateur de l'arbitrisme ${ }^{19}$ -, ne pouvaient être réduits au bullionisme étroit dont voulaient rendre compte les manuels d'histoire de la pensée économique ${ }^{20}$. Il convenait au contraire de souligner la profondeur de leur examen des causes de la «décadence » espagnole ${ }^{21}$, en recherchant dans leurs discours des informations concrètes sur l'économie et la société dans une période critique et des clefs d'interprétations qui, si elles n'étaient pas toujours originales, pouvaient être rapprochées de celles des Botero, des Bodin ou des Montchrétien. La réédition de nombreux textes d'arbitristes ${ }^{22}$ et les monographies 
consacrées à l'un ou l'autre d'entre eux ${ }^{23}$ obéissent à cet impératif. Mieux considéré, l'arbitriste l'est aussi dans les dictionnaires de l'espagnol courant, qui, plutôt que d'en faire un inventeur de nouveaux impôts, insistent sur sa bonne volonté et son désir d'alléger les charges des sujets - mais sans préjuger de son succès ${ }^{24}$.

Pour autant, le nom d'arbitriste n'a pas perdu sa connotation péjorative, et cette réhabilitation s'accompagne le plus souvent d'une distinction entre les auteurs de mémoires dignes d'attention et les autres. Le jugement de valeur est toujours présent, séparant, selon le mot de José-Luis Abellán, le «bon grain » de l'« ivraie »" Les meilleurs vont au-delà de l'expédient fiscal et financier et proposent de véritables réformes de la société espagnole qui s'enracinent dans une réflexion sur les causes économiques et sociales du déclin ${ }^{26}$. On les distingue des fous et des médiocres inventeurs d'expédients, souvent soupçonnés de préférer leur intérêt égoïste au bien commun : il semble aller de soi que la poursuite de l'intérêt individuel implique l'étroitesse de vues ${ }^{27}$. Aux premiers, on décerne le titre d'économiste afin de lever toute ambiguïté sur la qualité de leur pensée $^{28}$, qui relève de l'« économie politique ${ }^{29}$. Le mot «repúblico», récemment appliqué par Jean Vilar à Martin Gonzalez de Cellorigo ${ }^{30}$, présente l'avantage de ne pas être anachronique, l'historien reproduisant ici le jugement que Cellorigo portait sur luimême. Toutefois il ne résout pas entièrement la difficulté: on pourrait en effet l'appliquer à tous les autres écrivains (personne ne se reconnaissant arbitriste, nul ne devrait être appelé ainsi). Mais comment savoir, en l'absence de témoignage connu, dans quelle catégorie ses contemporains rangeaient Cellorigo?

La distinction opérée a amené à retenir les noms des

[...] escritores económicos que, desnudos de miras personales, nada piden para si, y abogan por el bien de los demás

dont parlait Pedro Rodriguez de Campomanes au XVIII ${ }^{e}$ siècle ${ }^{31}$. À quelques exceptions près, il s'agit des auteurs auxquels Manuel Colmeiro a accordé une place dans son histoire de la pensée économique, tout en dénonçant leur part de responsabilité dans la "décadence » de l'Espagne. Une telle distinction est éminemment problématique pour qui souhaite circonscrire le " groupe » des arbitristes. Les contemporains de Cervantès ne nous sont ici d'aucun secours au moment de déterminer qui est arbitriste et qui ne l'est pas: si chacun des auteurs de mémoires d'ordre économique ou politique se défend de l'être, les satires, pour leur part, dénoncent l'arbitriste en soi ou les arbitristes, mais ne donnent pas de noms. Qui est arbitriste ? Qui ne l'est pas? Ajoutons qu'à l'intérieur même de cette catégorie, comme l'a déjà signalé Jean Vilar ${ }^{32}$, les satiristes $d u$ XVII $^{\mathrm{e}}$ siècle ne distinguaient pas les bons des mauvais: comment alors ne pas craindre d'imposer à l'examen de l'arbitrisme des jugements de valeur étrangers à la perception de l'époque?

\section{Une absence d'identité sociale}

On peut légitimement être gêné par une description de l'arbitriste faite en termes essentiellement psychologiques. Dans les versions les plus caricaturales, l'arbitrisme serait la manifestation d'une humeur propre aux Espagnols, plus enclins que les autres à refaire le monde dès qu'ils s'attablent dans un café ${ }^{33}$. Il se situerait à mi-chemin entre la tare mentale et $l^{\prime} \ll$ esprit de la race $»^{34}$. Du coup, tout Espagnol est potentiellement $\operatorname{arbitriste}^{35}$. Un tel point de vue, qui fait de l'arbitrisme une manifestation intemporelle du caractère espagnol, interdit d'en faire l'histoire. 
14 Mais plus que ces jugements de valeur, ce qui nous importe ici, c'est l'absence de définition sociale de l'arbitriste. Être arbitriste n'est pas un statut, comparable à celui de marquis, d'hidalgo ou encore de secrétaire du roi. Tout au plus les satires anti arbitristes nous permettent-elles d'établir une catégorie par défaut: présenté comme un pauvre hère avide de gains et de récompenses, l'arbitriste n'est probablement ni un aristocrate ni un marchand-banquier; s'adressant essentiellement au roi ou aux Cortes, il est sans doute un homme de la ville, sinon de la cour. Définition bien imprécise! Pourtant, de façon paradoxale, les arbitristes ont souvent été traités comme un groupe séparé, et ce de deux manières.

D'une part, les tractations auxquelles se livrent les plus médiocres d'entre eux («l'ivraie») pour voir récompenser leurs inventions sont perçues comme les manifestations d'un vice (la cupidité). Cela implique que les procédures décrites ne sont pas normales : de là à penser que les arbitristes occupent tout au plus une place marginale dans l'administration d'un État décrit comme moderne, il n'y a qu'un pas. Tout se passe comme s'ils n'étaient que les vestiges d'une époque archaïque, voués à disparaître avec la rationalisation des institutions et les progrès de l'esprit humain. Telle est l'impression que donnent les comparaisons esquissées entre les proyectistas du XVIII ${ }^{e}$ et leurs ancêtres arbitristes ${ }^{36}$.

D'autre part, les meilleurs éléments (le "bon grain ») sont traités comme une sorte de groupe d'intellectuels ${ }^{37}$ ou de "penseurs ${ }^{38}$ moins empêtrés que les autres dans de vils conflits d'intérêts et susceptibles de s'élever au-dessus de la société pour élaborer une réflexion critique ${ }^{39}$. Cela affecte aussi bien l'analyse de leurs écrits que celle de leur action.

17 1. Leurs avis ${ }^{40}$ sont lus comme des traités théoriques d'économie politique: on y recherche une conception des échanges économiques, une définition de la richesse et de la valeur, une explication de la hausse des prix, ou encore « les antécédents d'une pensée économique à caractère scientifique ${ }^{41}$, notamment dans les monographies consacrées à l'un ou l'autre d'entre eux ${ }^{42}$. Levons toute ambiguïté : il ne s'agit pas de nier l'existence de cette dimension théorique. Ainsi, il ne paraît pas nécessaire de «forcer » les discours de Valle de la Cerda pour y trouver une expression de la théorie quantitative de la monnaie ou une conception tacitiste du roi d'un empire catholique. A l'instar de Jean Vilar, je crois que ce travail d'interprétation ${ }^{43}$ est indispensable si l'on veut saisir la signification du projet réformateur qui a fait passer le nom de cet arbitriste à la postérité ${ }^{44}$. En revanche, peut-être est-il anachronique de chercher dans les arbitristes des socialistes avant l'heure ${ }^{45}$ ou des chantres du libéralisme économique ${ }^{46}$ - en plein XVII ${ }^{\mathrm{e}}$ siècle -, ou encore d'en faire des théoriciens capables de se défaire de toute considération d'ordre moral et religieux dès lors qu'ils abordent les phénomènes économiques ${ }^{47}$ ?

18 Toutefois, une analyse précise de la pensée des arbitristes n'épuise pas leurs avis: la plupart des textes reconnus par les historiens comme des textes d'arbitristes sont avant tout des moyens d'action; leur objet premier n'est pas l'élaboration d'un système philosophique original, mais la défense d'un projet dont on réclame la mise en œuvre à brève échéance. Loin de se retirer dans une tour d'ivoire, les arbitristes participent par là aux conflits politiques du moment et tentent d'intervenir dans la prise de décision, qu'ils s'adressent aux ministres du roi ou aux Cortes.

19 2. Ils sont pourtant présentés comme des acteurs indépendants, ou du moins fondamentalement différents des ministres du roi, des procuradores aux Cortes ou des 
échevins des villes. C'est le cas lorsque Earl J. Hamilton étudie l'affrontement entre d'une part Philippe IV, qui ordonne en 1622 la création de « tresoryes » (erarios) et de monts-depiété tels que les conçoit la Grande Junte de Réformation (Junta Grande de Reformación), et d'autre part les villes et le clergé, qui dans leur immense majorité se refusent à appliquer la loi. L'auteur montre qu'à partir de 1623, le débat ne se tient pas uniquement dans l'enceinte des Cortes, intégrant à son analyse des avis de l'arbitriste Alberto Struzzi. Mais curieusement, il réserve pour un quatrième chapitre consacré à la postérité du projet de réforme financière étudié d'autres avis, remis ou non aux Cortes de 1623 et souvent contemporains de celles-ci ${ }^{48}$. D'un côté, le conflit politique; de l'autre, une réflexion critique plus distanciée. De façon comparable, Juan-Ignacio Gutiérrez Nieto analyse l'élaboration de la politique agricole au début du XVII ${ }^{e}$ siècle en séparant soigneusement trois entités : les Cortes, le Conseil de Castille et les arbitristes ${ }^{49}$. Sa démarche ne pose pas que des problèmes rhétoriques (elle l'oblige à se répéter). Elle présuppose aussi que les relations qui s'établissent entre ces trois entités ne sont que marginales dans cette histoire. Implicitement, les arbitristes sont conçus comme indépendants et cantonnés dans le monde des idées : ce n'est que par ce biais qu'ils peuvent influencer les débats, comme des intellectuels.

Dans ces deux cas, il est permis de s'interroger sur les critères qui autorisent à ranger tel arbitriste dans la catégorie des acteurs du débat (comme Alberto Struzzi) et tel autre dans celle des philosophes. Doit-on attribuer ces choix à un manque d'informations? Cela semble être le cas d'Earl J. Hamilton. Jerónimo de Ceballos et Juan de Urbina, dont l'historien américain ne traite que dans son chapitre IV, sont l'un échevin de Tolède, envoyé par la ville à la cour pour y faire des propositions fiscales ${ }^{50}$, l'autre secrétaire, probablement mandaté par Philippe IV aux Cortes ${ }^{51}$ - mais à quel titre le distingue-t-il de Struzzi, qui se rend aux mêmes Cortes? Il devient alors difficile d'arracher ces arbitristes aux affrontements politiques du moment.

21 Cependant, le fait que l'on étudie les discours des arbitristes plus que leur action, en les lisant comme des traités d'économie politique, obéit à une raison plus profonde : décrits comme des intellectuels qui n'entrent qu'incidemment dans l'arène politique, ils constitueraient un monde à part. Parler de la "pensée économique, politique et sociale des arbitristes », comme le fait Juan-Ignacio Gutiérrez Nieto, revient à postuler l'existence d'une sorte de «mouvement » arbitriste, conscient ou pas. José-Luis Abellán n'hésite pas à employer le terme ${ }^{52}$. Tous partageraient peu ou prou la même pensée et ceux qui n'étaient pas arbitristes ne feraient, au mieux, que la leur emprunter ${ }^{53}$; ce " mouvement » aurait son " précurseur », Luis de Ortiz ${ }^{54}$, et trouverait, son " antécédent » ${ }^{55}$ ou une sorte d'initiateur dans Cellorigo ${ }^{56}$ - Sancho de Moncada constituant le premier véritable représentant du genre ${ }^{57}$.

\section{Le facteur économique et ses limites}

L'apparition des arbitristes est historiquement datée. Les travaux de Jean Vilar, on l'a vu, permettent d'en situer la naissance entre la fin du règne de Philippe II et le début XVII siècle. L'apogée de l'arbitrisme serait contemporain des règnes de Philippe III et Philippe IV, coïncidant ainsi avec les difficultés financières suscitées par les guerres européennes (surtout à partir de 1621) ${ }^{58}$. La récente révision de l'historiographie traditionnelle du règne de Charles II (une période moins «décadente » qu'il n'y paraissait) conduit Juan A. Sánchez Belén à souligner la vivacité d'un arbitrisme plus 
tardif, celui du dernier tiers du XVII ${ }^{\mathrm{e}}$ siècle ${ }^{59}$. Pour ce qui est du XVIII ${ }^{\mathrm{e}}$ siècle, on s'accorde généralement à voir dans les proyectistas et leurs proyectos les héritiers des arbitristes et des arbitrios, suivant en cela les distinctions établies par José Múñoz Pérez ${ }^{60}$.

La coïncidence entre son apparition et le début de la «décadence " laisse penser que l'arbitrisme est seulement une conséquence de la crise économique qui affecte au moins une grande partie de la Castille au Siècle d'or ${ }^{61}$. Doit-on voir dans cette interprétation l'effet de la préférence accordée aux discours des arbitristes plutôt qu'à leur action? Puisqu'ils parlent avant tout des difficultés d'ordre économique, il parait logique d'attribuer leur apparition à la crise. Or, cette explication peut paraître insuffisante. En effet, des recherches récentes montrent que ladite crise n'est pas généralisée ${ }^{62}$. On est en outre tenté de souligner le décalage qui existe entre le discours des arbitristes, délibérément dramatique, et la réalité ${ }^{63}$. Par ailleurs, ces discours n'ont pas pour seul objet la situation des finances royales ou la crise agricole et démographique ${ }^{64}$. Enfin, la production arbitriste n'est pas rythmée par la seule situation économique, mais aussi par l'histoire politique. Ainsi, les pics de cette production coïncident avec l'effervescence des changements de règne $(1598,1621)$ ou de favori du roi $(1618,1643)^{65}$, qui peuvent donner aux arbitristes les plus critiques à l'égard de l'ancienne équipe dirigeante l'espoir d'être entendus ${ }^{66}$.

\section{Deux arbitrismes}

24 On pourrait conclure qu'il y a deux images de l'arbitrisme: l'arbitrisme-repoussoir, auquel on tente d'arracher les meilleurs esprits, en les taxant d'économistes, et un arbitrisme qui se confond avec un mouvement d'intellectuels. Ni l'une ni l'autre ne sont satisfaisantes.

La première repose sur une distinction entre des inventeurs vénaux et enfermés dans le court terme et des réformateurs désintéressés. Rien n'indique que cette distinction ait été faite par les hommes du XVII ${ }^{\mathrm{e}}$ siècle. La limite à établir entre les bons et les mauvais est pour le moins fluctuante : à partir de quel degré de complexité ou d'universalité de leurs projets deviennent-ils d'honorables réformateurs?

Quant à l'arbitrisme circonscrit à un petit nombre de réformateurs méritants, il vide de son sens le terme arbitrista, auquel on ne peut ôter sa connotation péjorative, comme l'a rappelé Jean $V{ }^{1}{ }^{6}{ }^{67}$. Cet arbitrisme a certes des frontières, celles d'un "groupe de penseurs » ou d'un «mouvement». Mais il est aussi difficile d'en définir la nature que pour le premier puisque les seules caractéristiques communes aux arbitristes sont celles de leur discours, dans sa forme et, dans une moindre mesure, dans ses objets.

Dans les deux cas, le regard porté sur l'arbitrisme n'est pas exempt d'anachronisme, soit que l'on suppose que la poursuite de l'intérêt personnel invalide la «pensée » des arbitristes, soit que l'on croie que seules les pensées complexes et originales sont dignes d'intérêt pour comprendre l'Espagne du Siècle d'or, soit encore que l'on en fasse a priori des êtres qui se situent en dehors de la société et de l'action politique.

Comment proposer une histoire plus complexe des arbitristes, en évitant les écueils qui viennent d'être évoqués? Il s'agit avant tout de les étudier sans les juger et de se demander si l'on peut comprendre l'arbitrisme autrement qu'en faisant de lui un mouvement de pensée. 


\section{À la recherche de l'arbitriste}

29 tenté de prendre pour point de départ une conception élargie des arbitristes, n'excluant ni ceux que Juan-Ignacio Gutiérrez Nieto appelle les arbitristes fiscaux, les inventeurs d'expédients à courte vue, ni les « économistes »- les Moncada, les Ceballos ou les Valle de la Cerda. Mais même ainsi, il demeure malaisé de délimiter les frontières du groupe des arbitristes. Quels critères retenir?

\section{L'arbitrisme n'est pas un mouvement}

La nature de leurs discours ne permet pas d'établir de discrimination pour deux raisons. La première est que, malgré les points communs signalés par Jean Vilar et Juan-Ignacio Gutiérrez Nieto, ce discours n'est pas unique, tous les arbitristes n'ayant, par exemple, pas la même conception des sources de la richesse. Juan-Ignacio Gutiérrez Nieto est d'ailleurs le premier à devoir distinguer les "agraristes" de ceux qui, comme les arbitristes de Tolède, donnent la primauté à la manufacture textile ${ }^{68}$. De la même façon, on peut opposer les Tolédans aux Sévillans et à quelques arbitristes intéressés par le commerce avec les Pays-Bas (Manuel López Pereira, Alejandro Lindo, Alberto Struzzi), défavorables à un protectionnisme commercial excessif ${ }^{69}$.

31 La seconde raison est que le discours arbitriste n'est pas l'apanage de ceux que l'on qualifie d'ordinaire d'arbitristes : on en retrouve les caractéristiques, telles qu'elles sont définies par Jean Vilar, sous la plume du comte-duc d'olivares ${ }^{70}$ ou dans des cédules royales $^{71}$. Il n'est pas rare non plus que des délégués des villes aux Cortes, à l'instar d'Hernando de Quiñones, procurador de León, se présentent à l'assemblée avec des discours préalablement écrits et construits comme ceux des arbitristes ${ }^{72}$. De la même façon, on peut montrer que les échevins de Burgos, Juan Alonso de Salamanca et Diego de Curiel, bons lecteurs des ouvrages de Miguel Giginta et de l'arbitriste Cristóbal Pérez de Herrera, s'en sont inspirés pour élaborer leurs discours lus au sein du collège municipal en $1600^{73}$. Ajoutons que si, dans ce dernier cas, il est possible d'identifier des plagiaires et des plagiés, en reconnaissant des arbitristes dans les seconds, rien ne nous autorise à affirmer qu'il en va toujours ainsi. Il est probable que les échevins et les ministres écrivent souvent comme les arbitristes, plutôt que d'en imiter un en particulier. Mais on ne saurait écarter un schéma inverse : peut-être les arbitristes, au moment d'écrire, ne font-ils qu'adopter les formes de pensée et les tournures communes à ceux qui se mêlent de décision politique, dans les Conseils, les villes, les juntes ou les Cortes? En l'absence d'une étude systématique des discours produits par ces différentes institutions - et non par les seuls auteurs de traités politiques - il serait imprudent de se prononcer.

32 Si l'on rappelle en outre qu'aucun arbitriste ne se reconnait comme tel, mais revendique d'autres appartenances (une ville d'origine, sa condition noble, les services rendus par lui et les siens), il devient extrêmement délicat de parler de «mouvement de penseurs » : les non arbitristes pensent aussi, et les affinités intellectuelles entre les arbitristes sont parfois ténues.

Les Cahiers du Centre de Recherches Historiques, 24 | 2009 


\section{Une catégorie en creux}

point de vue, il existe une ligne de séparation. L'usage commun oppose les ministres du roi ou les procuradores des Cortes aux arbitristes ${ }^{74}$. C'est semble-t-il ce que faisaient les contemporains : aucune des interventions d'Hernando de Quiñones aux Cortes de 1617-20 ne fut critiquée, alors même que l'on rappelait qu'il convenait de ne pas écouter d'arbitristas ( $1^{\text {er }}$ février 1618$)$, tout comme on l'avait déjà dit en 1588 . Quiñones n'était donc pas un arbitriste. Mais qu'est-ce qui le distinguait alors d'un Pérez de Herrera ou d'un Valle de la Cerda? Il paraît délicat d'invoquer leurs origines sociales respectives tant que l'on ne sait pas avec précision qui est arbitriste et qui ne l'est pas. En revanche, il est possible de parler de statut, si l'on se sert des clefs que nous donnent José Múñoz Pérez et Jean Vilar. Pour le premier, l'arbitriste se distingue du proyectista en ce qu'il remet des mémoires de sa propre initiative, alors que le proyectista est « fonctionnaire » du roi ${ }^{75}$. On aura l'occasion de revenir sur ces distinctions, contestables, mais retenons pour l'instant que l'arbitriste est perçu comme un individu qui n'a d'autre légitimité que celle qu'il s'accorde. Pour Jean Vilar, l'arbitriste est aux yeux des satiriques celui qui se mêle de ce qui ne le regarde pas, qui sort de son état en parlant du bien public et en voulant imposer de nouvelles taxes au peuple ${ }^{76}$. Autrement dit, il n'a pas d'autorité pour parler, contrairement à un procurador mandaté aux Cortes par sa ville, un échevin ou le membre d'un Conseil. On pourrait alors voir dans l'arbitrisme une "catégorie en creux", qui indique «ce que l'on n'est pas $»^{77}$ : être arbitriste, c'est surtout ne pas être échevin, favori du roi ou conseiller.

Aussi n'est-on point arbitriste en toutes circonstances. Don Baltasar Jiménez de Góngora, procurador de Cordoue aux Cortes de 1617-1620, propose le 28 août 1617 de mettre en place un impôt sur le sucre dont il est l'inventeur. L'ayant proposé à la Couronne en 1613, il avait obtenu une cédule lui assurant $4 \%$ des bénéfices en cas d'application de la mesure ${ }^{78}$, ce qui faisait de lui un arbitriste conforme à l'archétype. En 1617, c'est le procurador qui parle, et ses offres ne sont pas plus rejetées par ses collègues, pourtant réticents à l'égard des arbitristes, que celles d'Hernando de Quiñones.

Il reste que la frontière qui sépare arbitristes et non arbitristes n'est pas clairement marquée. Ainsi, les tentatives de mise en place du réseau de tresoryes et monts-de-piété imaginé par Luis Valle de la Cerda entre les règnes de Philippe II et de Philippe IV donnent lieu à un débat où interviennent plusieurs personnages dont il est malaisé d'établir le statut. Les avis en matière fiscale d'Agustín Álvarez de Toledo, auditeur d'une des Chambres des comptes (1581) puis membre du Conseil des Indes (1589-1601) ${ }^{79}$, furent constamment sollicités en dehors des organismes auxquels il appartenait, par des juntes ou par Mateo Vázquez, le secrétaire du roi. Álvarez de Toledo, alors, ne parlait pas en tant que membre du Conseil des Indes, mais comme spécialiste d'un projet d'impôt unique sur la farine et comme habile juriste, capable de justifier la suspension provisoire du privilège fiscal du clergé et des nobles ${ }^{80}$. Quoiqu'il n'ait jamais réclamé de droit d'avis, il considérait, tout comme les proches de Philippe II, que ses mémoires faisaient partie de ses services au roi, et le souverain insistait pour qu'on le remercie ${ }^{81}$. Doit-on, dans ces conditions, le considérer comme un arbitriste ou comme un ministre du roi ? Gaspar de Pons $^{82}$ suscite la même interrogation : cesse-t-il d'être arbitriste en entrant au Conseil des finances ? À l'autre extrémité de la période considérée, trois personnages figurent dans

Les Cahiers du Centre de Recherches Historiques, 24 | 2009 
les annales des arbitristes : Jerónimo de Ceballos et don Fernando de Toledo sont envoyés par la ville de Tolède à Madrid pour réclamer la mise en place d'une junte chargée d'examiner un certain nombre de propositions d'ordre fiscal et économique réunies par les autorités municipales ${ }^{83}$, tandis que Mateo Lisón y Biedma, échevin et procurador de Grenade, multiplie les mémoires hostiles à la politique du comte-duc ${ }^{84}$. Sont-ils d'emblée arbitristes, ou faut-il attendre que Ceballos publie, de sa propre initiative, son Arte real et que don Fernando de Toledo entre-apparemment sans mandat des autorités tolédanes - dans les juntes mises en place par Olivares pour les juger tels? Le cas de Lisón y Biedma est plus complexe puisque, s'il est momentanément désavoué par les Cortes, il continue d'être représentant de sa ville à la cour après les Cortes de 1621, chargé d'en défendre les intérêts et de contrôler l'activité des deux nouveaux délégués aux Cortes ${ }^{85}$.

\section{Vers une définition}

À l'intérieur de ces limites, il n'est pas exclu d'envisager une étude prosopographique de ceux qui se font arbitristes à un moment donné de leur vie. Elle tiendrait compte du statut des individus concernés, de leur trajectoire, des raisons pour lesquelles ils deviennent arbitristes, de leurs moyens d'action et des relations sur lesquelles ils peuvent compter pour parvenir à se faire entendre des Conseils, des juntes ou des Cortes. Une analyse comparable a été menée sur les donneurs d'avis de France par Françoise Bayard, à partir des avis conservés par le Conseil royal ${ }^{86}$. En Espagne, il conviendrait d'ajouter aux avis reçus par le Conseil des finances ceux adressés aux autres Conseils, aux juntes, aux Cortes, aux secrétaires ou aux conseillers les plus influents ${ }^{87}$.

Pour l'instant, en l'absence d'une synthèse qui exigerait probablement un effort collectif, on peut seulement avancer que les arbitristes sont issus d'un éventail relativement large de la société espagnole. Ce ne sont pas seulement des gens de robe, mais aussi d'anciens soldats, des marchands, des religieux, des médecins, des membres de l'administration royale ${ }^{88}$. Leurs relations avec les financiers qui traitent avec l'administration des finances royales sont plus mal connues, peut-être sous-estimées parce que l'on a délaissé les auteurs d'expédients financiers ou fiscaux à très court terme.

La carrière de nombre de ces arbitristes dépend étroitement des services rendus au roi : c'est du moins ce qui ressort de l'étude de ceux qui interviennent dans les débats sur les tresoryes et monts-de-piété de Valle de la Cerda ${ }^{89}$. Ils sont comptables de l'armée et des Conseils (Luis Valle de la Cerda, Juan López de Ugarte, Antonio de Rojas), secrétaires de membres de la famille royale (Alberto Struzzi), vétérans des Flandres, mi-espions misoldats (Luis Valle de la Cerda, Pierre d'Oudegherst, Ramón Ezquerra, Juan López de Ugarte), médecin du roi (Cristóbal Pérez de Herrera). D'autres, qu'ils soient marchands (Tomás de Cardona, Manuel López Pereira, Damián de Olivares) ou juristes (Pierre d'Oudegherst, Martín González de Cellorigo ou Gerardo Basso) ne font pas davantage partie de ceux qu'Olivares appelait les puissants (poderosos) : les services rendus au roi peuvent leur procurer une reconnaissance sociale qu'ils ne trouveraient peut-être pas dans leur ville d'origine. Rien d'original ici : Françoise Bayard montre que les donneurs d'avis de France sont, dans leur grande majorité, des gens du roi (des officiers de justice et des secrétaires $)^{90}$. Cette dépendance à l'égard du monarque pourrait permettre de comprendre pourquoi les arbitristes insistent tant sur sa capacité à transformer les mœurs ou à modifier l'activité économique ${ }^{91}$. En même temps, le fait de ne pas faire partie des puissants peut expliquer que nombre d'arbitristes ne soient pas gênés par des 
réformes qui visent à briser le monopole du pouvoir économique et social de ces derniers et qu'ils défendent une conception de l'honneur moins traditionnelle ${ }^{92}$.

\section{Au cœur du système politique} un discours théorique, mais aussi y déceler la défense d'intérêts concrets, ceux d'une région ou ceux de groupes sociaux (les marchands-fabricants de textile castillans, les négociants sévillans, les agriculteurs castillans). Ceci, sans qu'il soit nécessaire d'en faire les porte-parole d'un seul groupe, comme le serait une éventuelle bourgeoisie marchande. Prenons une fois de plus l'exemple utilisé tout au long de cet article : si Valle de la Cerda prétend, grâce à la mise en place de tresoryes et de monts-de-piété, favoriser l'activité des grands marchands, il ne reçoit pas pour autant leur appui. La plupart du temps, ceux-ci ne prennent pas part aux controverses sur les tresoryes. Quand ils le font, à la fin $\mathrm{du} \mathrm{XVI}^{\mathrm{e}}$ siècle et au début du XvII ${ }^{\mathrm{e}}$, leur intervention est celle d'un créancier du roi, d'un échevin de Burgos ou d'un marchand hanséate désireux de participer au financement du commerce américain ${ }^{93}$, plutôt que celle d'un marchand fier de l'être. Au début du règne de Philippe IV, ils s'opposent - au moins à Séville - à la mise en place de tresoryes.

2. Les moyens d'action des arbitristes sont loin d'être atypiques, même s'ils en ont moins que d'autres (les poderosos). Tout comme les conseillers du roi à la cour et les corrégidors dans les villes, ils s'appuient sur leur parentèle, ils ont recours à des protecteurs et à des compatriotes résidant à la cour et n'hésitent pas à rejoindre la faction la plus puissante $\mathrm{du}$ moment. John $\mathrm{H}$. Elliott souligne ainsi que les projets de réforme des proches d'Olivares vont de pair avec la mise à l'écart des clients du dernier favori de Philippe III. Valle de la Cerda est particulièrement habile à cet égard. A la fin $\mathrm{du} \mathrm{XVI} \mathrm{e}^{e}$ siècle, il trouve l'appui de son patron, don Juan de Idiáquez (dont il se dit la créature), ainsi que de fray Diego de Chaves, Pedro Moya de Contreras, Agustín Alvarez de Toledo, Jerónimo Gassol, tous liés à la faction alors dominante à la cour ${ }^{94}$. En 1599 , ne pouvant plus compter sur Idiáquez, il recherche et gagne la faveur du duc de Lerme, favori du nouveau roi, Philippe III ${ }^{95}$.

3. Il n'est pas certain que les satires anti-arbitristes soient l'expression d'une pensée majoritaire. Il conviendrait d'évaluer la place occupée par les arbitristes dans la littérature. On remarquera seulement ici, à l'instar de Jean Vilar, ${ }^{96}$, qu'ils sont loin d'y occuper le premier plan. Ainsi, dans la galerie des personnages grotesques de Molière, on ne rencontre que deux donneurs d'avis (dans Les Fâcheux). Chez Lope de Vega, Jean Vilar n'a repéré un seul arbitriste. Contrairement à ce qu'affirme José Múñoz Pérez ${ }^{97}$, jaloux, entremetteuses et maris trompés accaparent toujours le devant de la scène.

Les Cahiers du Centre de Recherches Historiques, 24 | 2009 

ce point de vue, les arbitristes se distinguent mal des proyectistas étudiés par José Múñoz Pérez. Le Conseil des finances, par exemple, conserve des mémoires d'arbitristes sur lesquels sont portés la date de réception du texte, le nom du ministre auquel a été transmise l'affaire, et éventuellement la décision prise ${ }^{98}$. La plupart des juntes consacrées à des questions financières ou fiscales travaillent à partir d'arbitrios et n'hésitent pas à entendre leurs auteurs ${ }^{99}$, les règles qui doivent régir le traitement des mémoires reçus étant parfois couchées sur le papier ${ }^{100}$. Quant aux Cortes, si les arbitristes y sont dénoncés à deux reprises en un demi-siècle $(1588,1618)$, l'élaboration de la politique fiscale à suivre à partir d'avis venus de l'extérieur parait être la règle à partir du moment où c'est le Royaume qui définit les conditions du servicio de millones. Pendant la session de 1617-1620, les délégués des villes consacrent ainsi plusieurs mois à réviser les arbitrios examinés au cours des précédentes réunions, relisant les actes des Cortes, ainsi que les nouveaux mémoires reçus, avant de choisir les moyens par lesquels seront prélevés les nouveaux millones. Francisco-José Aranda Pérez a enfin montré que les arbitristes ne s'adressaient pas seulement aux centres de pouvoir de la cour : en 1618, c'est le corps de ville de Tolède qui sollicite le tribunal de l'Inquisition, le chapitre ecclésiastique, les ordres religieux, l'université et les jurados pour réunir les avis qu'il veut adresser au roi et aux Cortes ${ }^{101}$. De la même façon, la ville de Madrid reçoit des arbitrios et les rémunère ${ }^{102}$.

En outre, en Espagne comme en France ${ }^{103}$, on traite souvent et sans honte avec les arbitristes, leur accordant les cédules qui leur garantissent un «droit d'avis » au cas où leur projet serait mis à exécution. On se souvient de l'exemple de Juan Gómez de Bedoya, étudié par Juan-Ignacio Gutiérrez Nieto ${ }^{104}$. Dans sa thèse consacrée aux arbitrios "monétaires", Helena Garcia Guerra a relevé de nombreuses cédules de privilèges accordées aux arbitristes ${ }^{105}$.

\section{Entre intérêt et idéologie}

À cet égard, il conviendrait peut-être de réviser le jugement porté sur l'âpreté au gain des arbitristes, censée affecter les plus médiocres. Le Flamand Pierre d'Oudegherst, initiateur du projet des tresoryes, demande à Philippe II de lui accorder un cinquième des gains au vu d'un court résumé de son projet ; ce n'est qu'après l'avoir obtenu qu'il envoie à Madrid une longue description du système ${ }^{106}$. Quant à Valle de la Cerda, il a passé sa vie à réclamer une charge de secrétaire en récompense de ses services. Aux services d'agent plus ou moins secret et de comptable de la Sainte Croisade, se mêlent ceux du donneur d'avis en matière d'État et de finance ${ }^{107}$.

Il n'est pas nécessaire de voir ici une contradiction interne entre l'intérêt égoïste et des pensées plus nobles. Deux raisons à cela. D'abord, l'intérêt est justifié : tout service mérite récompense dans ce que l'on a parfois appelé une "économie de la grâce » ${ }^{108}$. Ni Oudegherst ni Valle de la Cerda n'ont le sentiment que leurs demandes sont illégitimes. Leurs protecteurs leur reconnaissent le droit d'être récompensés: le roi remercie chaleureusement Oudegherst et promet de lui offrir un dixième des bénéfices si le projet est nouveau ${ }^{109}$; don Juan de Idiáquez, patron de Valle de la Cerda, s'engage à obtenir pour lui les grâces demandées ${ }^{110}$, engagement que sa créature ne manque pas de lui rappeler le moment venu ${ }^{111}$. En second lieu, la poursuite de leur intérêt individuel n'invalide pas la pensée de ces deux arbitristes. Fabián Estapé, si peu clément avec les arbitristes, a 
reconnu l'importance de celle d'Oudegherst ${ }^{112}$; pour sa part, Valle de la Cerda est plus souvent taxé d'économiste que de vil inventeur de chimères.

Ainsi, il parait naï d'opposer de purs réformateurs à des acteurs trop intéressés pour produire une pensée sur la société. La signification de l'action des arbitristes n'est pas univoque: on peut admettre que s'y mêlent les fins personnelles, la défense d'intérêts sectoriels, et ce que l'on a appelé au xixe siècle l'idéologie.

\section{Les arbitristes dans l'administration traditionnelle}

La place accordée aux arbitristes dans l'élaboration de la politique financière ou fiscale, via les juntes, les ministres influents ou les Cortes, invite à proposer une explication structurelle du phénomène. Ils ne sont pas uniquement l'effet de la crise économique, même s'il est indéniable qu'ils apparaissent avec elle. Loin de constituer un élément marginal dans l'administration royale ou dans les négociations entre le roi et les villes, ils semblent omniprésents. Il serait intéressant de le mesurer, en observant de près les canaux par lesquels leurs avis parviennent jusque sur la table des Conseils ou des secrétaires du roi et en évaluant la part des arbitrios qui reçoivent un privilège, puis la part des arbitrios effectivement appliqués. Travail, une fois de plus, qui demanderait la collaboration de plusieurs chercheurs.

50 L'étude de la gestation de telle ou telle réforme constitue un autre angle d'approche. Elle est généralement le fait d'historiens dont l'objet premier n'est pas l'histoire de l'arbitrisme $^{113}$. Ce point de vue conduit à souligner l'influence des arbitristes. N'y voyons pas une originalité péninsulaire : outre Pyrénées, le recours aux donneurs d'avis est l'un des moyens normaux de la recherche de fonds, à tel point que des secrétaires du Conseil royal sont payés pour résumer les avis reçus ${ }^{114}$. Tout porte donc à croire que les arbitristes, loin d'être un résidu archaïque dans une administration qui serait par ailleurs parfaitement rationnelle et impersonnelle, constituent l'un des rouages importants des finances de l'époque. Cela ne paraît guère étonnant: les historiens des finances ont montré que leur fonctionnement reposait sur la collaboration avec des tiers, des fermiers, des partisans (asentistas) ou des villes qui se chargeaient de lever leurs propres impôts. Aussi, est-ce probablement avec raison que la littérature satirique juge les arbitristes dangereux pour le contribuable, les assimilant parfois aux fermiers ou aux partisans, plus souvent décriés. De manière plus générale, on ne peut s'étonner de voir intervenir les arbitristes dans une administration « traditionnelle » - au sens wébérien - qui sélectionne ses membres en fonction de leur condition, des services de leurs ancêtres ou des recommandations de patrons puissants autant que de leurs capacités, et qui établit entre chacun d'eux et le roi une relation fondée sur le service et la récompense. Leur expérience et l'appui d'un protecteur peuvent suffire à les rendre acceptables dans une junte et leurs services sont aussi dignes de rétribution que ceux d'un soldat ou d'un Conseiller.

\section{Projets réformateurs et négociation politique}

51 Mais les arbitristes ne sont pas seulement des conseillers techniques. La longue révision par les Cortes en 1618 des arbitrios déjà examinés auparavant nous invite à ne surtout pas les cantonner dans le rôle d'experts des finances et de l'économie pour l'administration royale et les Cortes ou encore dans le rôle de courtiers des banquiers ou des fermiers des impôts auprès du roi. Le recours aux arbitristes doit être replacé dans une stratégie 
politique, qui fait de leurs projets de réforme ou de leurs expédients fiscaux et financiers autant un moyen qu'une fin. C'est précisément après que le roi a réclamé un nouveau servicio (29 mai 1617) que les délégués des villes aux Cortes entreprennent de relire tous les arbitrios étudiés au cours des années précédentes (à partir du 2 juin 1617). L'un d'entre eux retient tout particulièrement leur attention : le réseau de tresoryes imaginé par Valle de la Cerda, dont l'application figurait au nombre des conditions du servicio de los 18 millones voté en 1601. Le traité dans lequel Valle de la Cerda le décrivait est aussitôt publié, pour la deuxième fois, aux frais des Cortes (1618). Mais curieusement, une fois les conditions du nouveau servicio de millones établies, à l'avantage des dix-huit villes représentées aux Cortes ${ }^{115}$, il n'est plus question de fonder de tresoryes. Les villes, qui depuis 1601 sont restées parfaitement indifférentes au projet de Valle de la Cerda, quoiqu'elles aient signé le contrat du servicio de 1601 avec toutes ses conditions, ne sont pas consultées cette fois. Dans le même temps, la plupart des arbitrios examinés sont délaissés. Tout se passe comme si la mention des tresoryes n'avait constitué qu'un moyen de pression sur le roi dans la négociation du nouvel impôt ${ }^{116}$.

52 La Couronne fait parfois un usage comparable des projets des arbitristes. On pourrait prendre pour exemple les annonces répétées de révision des comptes des banquiers du roi et des procédures judiciaires mises en œuvre à l'occasion des suspensions de paiements, devenues presque annuelles à la fin du XVII ${ }^{e}$ siècle. Ce qui paraît se dessiner, c'est la réforme des pratiques financières que réclament les arbitristes depuis un siècle : les partisans disparaîtraient ou ils seraient étroitement contrôlés par le Conseil des finances. On en finirait avec la gabegie! De fait, Carmen Sanz Ayán montre que les menaces sont rarement mises à exécution, sauf de façon partielle ${ }^{117}$. On négocie avec les partisans les plus puissants, ceux qui sont en mesure de concéder de nouveaux crédits au roi. Les décrets de suspension des paiements, comme les chambres de justice en France ${ }^{118}$, sont, plutôt qu'une fin en soi, un moyen de pression dans la négociation de nouveaux contrats et une opération destinée à confirmer, aux yeux des sceptiques, la moralité de la Couronne. La politique de récupération de recettes fiscales injustement accaparées par les nobles, ce désengagement du patrimoine aliéné si cher aux arbitristes ${ }^{119}$, peut être comprise de la même façon : souvent, le procès est provisoirement suspendu, une fois que le noble indélicat a accepté de verser une composition à la Couronne ${ }^{120}$.

On ne saurait en déduire que les arbitristes sont dupes des plus puissants, ministres du roi ou échevins. Les précautions oratoires qu'ils prennent pour annoncer les mesures les plus radicales - dans le cliché littéraire, l'insistance sur la «facilité » d'exécution de leur projet - et la souplesse avec laquelle certains d'entre eux modifient leurs propositions en fonction des objections qui leurs sont faites ${ }^{121}$ indiquent qu'ils sont parfaitement conscients du fait qu'il est impossible d'appliquer en tous points la politique qu'ils défendent.

$\mathrm{Au}$ fond, on ne saurait s'en tenir à une définition purement négative des arbitristes. Ils constituent certes une catégorie " en creux » puisque ni leurs origines sociales ni leurs projets ne suffisent à les identifier. Leur statut social est presque un non-statut : il leur manque l'autorité qui leur permettrait d'échapper à la vindicte des satiriques. Toutefois, ils se définissent aussi positivement par l'espace qu'ils occupent dans un système politique, entre le roi et les différents corps de la société, entre la cour, les Cortes et les villes. Sans prétendre en faire le cœur de ce système, on peut affirmer qu'ils jouent un rôle essentiel dans la décision et la stratégie politique : ils sont présents dans les juntes et leurs avis s'empilent sur les bureaux des Cortes, des secrétaires du roi et des Conseils. 

indépendant, décrit par Weber ${ }^{122}$. Leurs relations privées comptent autant, sinon plus, que leurs compétences objectives. Leur rapport au roi est conçu en termes de services et de récompenses, de protection bienveillante et de gratitude, et ils confondent leur intérêt propre avec le bien public. Quant au roi, il semble ne pas pouvoir se passer d'eux, qu'ils soient ou non ses officiers, sollicitant constamment leurs avis. L'annonce d'une réforme ou d'un nouvel impôt ne lui assurant jamais le concours automatique des groupes les plus puissants (les grands financiers, le clergé, ou les pouvoirs municipaux), il se servira des projets des arbitristes pour faire pression sur ses interlocuteurs et obtenir des contreparties. Nous avons vu que les Cortes et quelques-unes des villes qui y étaient représentées pouvaient développer les mêmes stratégies.

Plutôt que d'exclure les arbitristes du système politique ou d'en faire les vestiges d'une époque plus ancienne, en les décrivant comme des personnages atypiques, soit des rêveurs risibles ou dangereux, soit des philosophes enfermés dans leur tour d'ivoire, on a préféré en faire ici des acteurs à part entière de ce système. Ceci conduit à souligner, après d'autres ${ }^{123}$, le caractère « traditionnel » de l'administration et plus généralement de la société dans laquelle ils vivent. En même temps, cela suppose que l'on ne traite pas les arbitristes seulement comme des écrivains. Savoir comment leurs textes ont été lus et utilisés-qu'on les ait compris ou non-est aussi important que de saisir leur signification.

\section{NOTES}

1. Literatura y economía ; la figura satírica del arbitrista en el Siglo de Oro, Revista de Occidente, Selecta, 48, 1973.

2. Juan-Ignacio Gutiérrez Nieto, «El pensamiento económico, político y social de los arbitristas », Historia de España Menéndez Pidal, El siglo del Quijote (1580-1680) : Religión, filosofía, ciencia, Ramón Menéndez Pidal (dir.), Madrid, Espasa Calpe, 1986, p. 235-354.

3. On les cite dès que l'on évoque les arbitristas et l'arbitrismo. Quelques exemples récents, sans prétendre à l'exhaustivité : Pedro Álvarez de Miranda, « Proyectos y proyectistas en el siglo XVIII español », Boletín de la Real Academia Española, LXV, cuaderno CCXXXVI, 1985, p. 409-30; JoséAntonio Álvarez Vázquez, « Arbitristas españoles del siglo XVII », Cuadernos Hispano-Americanos, $\mathrm{n}^{\circ}$ 334, 1978, p.55-75; Sará Almarza, «Los vocablos «arbitrio» y «arbitrista en el Nuevo Mundo ", Cuadernos hispanoamericanos, n 374, agosto de 1981, p. 421-29 ; Mercedes Barat, «Un texto arbitrista del siglo XVII : el memorial de Ángel Manrique ", Cuadernos de historia moderna y contemporánea, $\mathrm{n}^{\circ}$ 2, 1981, p. 105-25; Benjamín Gonzalez Alonso, «El Conde Duque de Olivares y la administración de su tiempo », La España del Conde Duque de Olivares. Encuentro internacional sobre la España del Conde Duque de Olivares celebrado en Toro los días 15-18 de septiembre de 1987, Universidad de Valladolid, 1990, p. 274-311; Frédérique Langue, « De moralista a arbitrista : Don Francisco de Ibarra, obispo de Venezuela (1798-1806). Recopilación documental. », Suplemento de Anuario de Estudios Americanos. Sección Historiografia y Bibliografia, t. XLIX, nº 1, Séville, 1992 ; Manuel M. Martin Galán, «La política de la privanza », Historia 16, n 204, 1993, p. 46-52 ; Maria-Luisa Martínez de Salinas, «Contribución al estudio sobre los arbitristas. Nuevos arbitrios para las 
Indias a principios del siglo XVII ", Revista de Indias, vol. L, nº 188, 1990, p.161-69; Giovanni Stiffoni, « Tematiche dell'arbitrismo politico e articolazione dello spazio del potere nella Spagna del dispotismo illuminato », Revista de historia moderna. Aspectos de la administración española del siglo XVIII, n 13-14, 1995, p. 13-29.

4. Jean Vilar les a employés jusqu'à une date récente: "de l'arbitrisme au rêve eugénique", «l'arbitrisme majeur espagnol», «les arbitristes affichés dispersés dans l'orbite du monde hispanique », «le pragmatisme arbitriste ». Voir «Vers Campanella: substituts pragmatiques espagnols à quelques fonctions utopiques fondamentales", Colloque franco-hispanique: les utopies dans le monde hispanique, Casa de Velázquez, Madrid, 1990, p. 200, 203, 204. On les retrouve sous la plume d'autres historiens. À titre d'exemples: Jean-Frédéric Schaub, «L'État quotidien entre arbitrisme et révolte, la gabelle au temps du comte-duc d'Olivares ", Recherches sur l'histoire de l'État dans le monde ibérique, Presses de l'École normale supérieure, Paris, 1993, p. 21-50 ; Michel Cavillac, Gueux et marchands dans le Guzmán de Alfarache (1599-1604). Roman picaresque et mentalité bourgeoise dans l'Espagne du Siècle d'or, Institut d'études ibériques de l'université de Bordeaux, Bordeaux, 1983, p. 265.

5. Sur leur fortune historiographique depuis le xviII ${ }^{\mathrm{e}}$ siècle : Jean Vilar, Literatura, introduction et L'« arbitrista » malgré lui. La vie et les écrits du Licencié Cellorigo (1565 ?-1630 ?), Thèse de doctorat, université de Paris IV, sous la direction de Jean-Pierre Etienvre, novembre 1996, introduction ; Michael D. Gordon, introduction à Pedro Fernández Navarrete, Conservación de monarquías y discursos políticos (1626), Instituto de Estudios Fiscales, Madrid, 1982 ; José Antonio Àlvarez Vázquez; "Arbitristas españoles del siglo XVII », 1978. Je reviens sur la question dans l'introduction de ma thèse consacrée à l'arbitriste Luis Valle de la Cerda, voir la version publiée, Réformer les finances espagnoles au Siècle d'or : le projet Valle de la Cerda, Clermont-Ferrand, Presses universitaires Blaise-Pascal, 2000 ; Les articles et ouvrages relatifs à l'Espagne moderne qui comportent les mots arbitrisme, arbitriste, arbitrismo, arbitrista se comptent par dizaines pour les trois dernières décennies $\mathrm{du} \mathrm{xx}^{\mathrm{e}}$ siècle. Plutôt que d'en rappeler la liste exhaustive (que l'on trouvera dans les bonnes bases de données bibliographiques), je signalerai ici les tendances les plus significatives de la recherche.

6. Jean Vilar, Literatura, chap. I à III.

7. Manuel Colmeiro Penido, le premier à consacrer de longs développements aux arbitristes au milieu du XIX ${ }^{\mathrm{e}}$ siècle, ne parlait pas, à ma connaissance, d'arbitrismo (Biblioteca de los economistas espanoles de los siglos XVI, XVII y XVIII, Madrid, Publicaciones de la Real Academia de Ciencias Morales y Políticas, 1953-54, $1^{\text {re }}$ éd. : 1861 ; Historia de la economía política de España, Biblioteca Política Taurus, Madrid, 1965, $1^{\mathrm{e}}$ éd. : 1863. Si le Diccionario de autoridades (1726-39), celui de Terreros (Diccionario castellano con las voces de ciencias y artesy sus correspondientes en las très lenguas francesa, latina é italiana, 1786) et les dictionnaires plus récents de María Moliner et de l'Académie Royale comportent des définitions d'arbitrista, le mot arbitrismo n'y figure pas. On le rencontre dans le Diccionario de historia de España dès 1952 ; Germán Bleiberg (dir.), Revista de Occidente, Madrid, rééd. 1968, article «Arbitrismo » de Fabián Estapé ; Jean Vilar l'utilisait en 1973 sans commentaire (Literatura, p. 18, 36, 44, 49, 59.

8. John H. Elliot, «Introspección colectiva y decadencia en España a principios del siglo XVII », 1977, p. 193-223, Podery sociedad en la España de los Austrías, Barcelone, 1982, chap. VI.

9. En 1588 on condamne l'excessive confiance accordée aux arbitrios (Jean Vilar, Literatura, p. 36-43). Le $1^{\text {er }}$ février 1618 , le procurador don Luis de Castilla invite ses pairs à ne pas écouter les " arbitristas ", leur rappelant les résolutions prises par les procuradores en 1588 (ACC, vol. XXXI, p. 246). Jean Vilar a précisé les multiples significations du terme arbitrio. Il désigne-entre autres - les remèdes ou moyens préconisés ou, dans une acception péjorative, les expédients financiers ou fiscaux proposés par les arbitristes ou mis en place par les financiers du roi ( Literatura, p. 23-31). 
10. Jean Vilar signale que c'est la traduction donnée dans une version française du Coloquio de los perros de Cervantès en 1615 (Literatura, p. 55). Elle est reprise dans le Tesoro de las dos lenguas española y francesa de César Oudin, Paris, Ediciones hispano-americanas, 1968, fac-similé de l'éd. de 1675 et dans le dictionnaire de Terreros un siècle plus tard (note 7). Pour une comparaison avec la France on se reportera à la synthèse de Françoise Bayard, Le Monde des financiers au XVII ${ }^{e}$ siècle, "Bibliothèque scientifique ", Paris, Flammarion, 1988, et aux indications données par Andrée Chauleur, "Le rôle des traitants dans l'administration financière de la France de 1643 à 1653 ", XVII ${ }^{\mathrm{e}}$ siècle, $\mathrm{n}^{\circ} 65,1964$, p. 20 ; Roland Mousnier, La Vénalité des offices sous Henri IV et Louis XIII, Paris, Presses universitaires de France, 1971, p. 150-51.

11. Les juntes sont des groupes d'individus issus ou non de l'administration royale, réunis pour traiter d'un sujet précis. Souvent choisis en vertu de leur expérience ou de leur relation personnelle au favori du roi, ils travaillent en dehors du cadre des Conseils existants. La durée des juntes, leurs compétences juridiques, etc., sont variables : Juan-Francisco Baltar Rodriguez, Las Juntas de Gobierno en la Monarquia Hispànica, Madrid, Centro de Estudios Políticos y Constitucionales, 1998.

12. Juan-Ignacio Gutiérrez Nieto a publié un mémoire de l'arbitriste Juan Gómez de Bedoya dans lequel ces démarches sont mises en évidence («El pensamiento», p. 241); Fred Bronner, «Tramitación legislativa bajo Olivares. La rédaction de los arbitrios de 1631 », Revista de Indias, XLI, $\mathrm{n}^{\circ} 165-66,1981$, p. 413.

13. Chacun de ces arbitristes a fait l'objet de nombreux travaux. On se reportera à ceux de Michel Cavillac, «Noblesse et ambiguïtés au temps de Cervantès : le cas du docteur Cristóbal Pérez de Herrera (1556 ?-1620) », MCV, n 11, 1975, p. 177-21 ; Juan-Ignacio Gutiérrez Nieto, « El pensamiento " pour le cas de Cristóbal Pérez de Herrera et à ceux de Jean Vilar pour Martin Gonzalez de Cellorigo et Sancho de Moncada, L'«arbitrista» malgré lui; éd. de Sancho de Moncada, Restauration politica de España, Instituto de Estudios Fiscales, 1974. Voir également leurs bibliographies. Luis Valle de la Cerda est au centre de ma thèse.

14. Jean Vilar analyse les discours des arbitristes, tels qu'ils sont écrits et tels qu'ils sont perçus par dramaturges et romanciers (Literatura). Les définitions des dictionnaires, jusqu'au début du $\mathrm{xx}^{\mathrm{e}}$ siècle, se font l'écho de la satire (Diccionario de Autoridades; Terreros, Diccionario castellano).

15. À propos de Luis Valle de la Cerda, Manuel Colmeiro écrit : Cae el escritor en el yerro común a los arbitristas de considerar su pensamiento como único remedio a los males de España, Biblioteca, $\mathrm{n}^{\circ} 384$, p. 149-50; plus généralement: En dos cosas se manifiesta el carácter atrevido y jactancioso de los arbitristas : en su presunción de anunciar al mundo ruidosas verdades y de pasar por mensajeros de Dios para mostrar el camino de la redención de España, y en su vanidad de curar de un golpe y con un solo medicamento todos los males de la república, Historia, t. II, p. 586.

16. C'est le cas de Fabián Estapé, auteur en 1952 d'une notice sur l'arbitrisme (note 7) : La inmensa mayoría de los proyectos que se redactaron no eran más que lucubraciones fantásticas, reflejo de la búsqueda de una piedra filosofal que debería proporcionar al erario una fuente inagotable de recursos y al país el retorno a pasados esplendores. [...] La consideración y el conocimiento de nuestro pasado económico ganará en precisión cuando, olvidando los absurdos remedios (sustituir las mulas por bueyes en las faenas agrícolas, como proponía Arrieta ; establecer Montes de Piedad, como quería Valle de la Cerda; crear una compañía universal de fábricas y comercio o hacer navegables todos los ríos de España, como pretenden tantos otros), la atención se centre en todos los males que los arbitristas quisieron curar; Evaristo Correa Calderón, auteur d'un catalogue des écrits d'arbitristes et réformateurs des $\mathrm{XVI}^{\mathrm{e}}$ $\mathrm{XVIII}^{\mathrm{e}}$ siècle, tout en soulignant l'intérêt de ces textes, reproduit le cliché, parlant de gentes de desatinada fantasia, entre los que abundaban los arbitristas, inventores y proyectistas, los moralistas, los imaginadores et aussi des utopistas que pretendían el logro de la paz perpetua y la cuadratura del círculo ; l'objet du catalogue est de dresser un amplio horizonte de temas e inquietudes, que van desde el puro arbitrismo (entendiendo el vocablo como invención delirante) hasta los escritos más serios y graves, 
Registro de arbitristas, economistas y reformadores españoles (1500-1936), Fundación Universitaria Española, 1982, p. 19 et 25.

17. Traitant du projet des tresoryes (erarios) et monts-de-piété de Luis Valle de la Cerda, Pedro Schwartz reproche aux contemporains de l'arbitriste d'ignorar las razones de la liquidez y el precio del tiempo, no entender la esencia de la institución de la propiedad privada y los beneficios que de ella se derivan para la sociedad, «El rechazo de los erarios por las Cortes de Castilla en la primera mitad del siglo XVII ", José Manuel de Bernardo Ares et Enrique Martínez Ruiz (éd.), El municipio en la España moderna, Cordoue, Universidad de Córdoba, 1996, p. 355-79; À propos de l'usure : En resumen, ; que el lucro cesante se convertía por arte teológica en daño emergente! Es pena que aún estuviera tan lejos el descubrimiento de la noción de coste de oportunidad, «Juntar erarios y montes de piedad : un arbitrio barroco ante las Cortes de Castilla ", Revista de Historia Económica, año XIV, $1996, n^{\circ} 1$, p. 78 et 65 . Soyons compatissants.

18. José Larraz Lopez, La época del mercantilismo en Castilla (1500-1700), Madrid, 1943 ; Earl J. Hamilton, «La decadencia española en el siglo XVIII », El florecimiento del capitalismo, Revista de Occidente, Madrid, 1948; Pierre Vilar, «Les primitifs espagnols de la pensée économique: quantitativisme et bullionisme ", Mélanges offerts à Marcel Bataillon, 1962 ; Jean Vilar, Literatura ; John H. Elliott, « Introspección ».

19. Manuel Fernández Álvarez, «El memorial de Luis de Ortiz », Anales de Economía, vol. xVII, $\mathrm{n}^{\circ}$ 63, 1957, p. 101-200 ; Jean Vilar, Literatura, p. 44 ; Sará Almarza, «Los vocablos »; Juan-Ignacio Gutiérrez Nieto, «El pensamiento », p. 247, 250 ; Henry Kamen, Vocabulario básico de la historia moderna, Barcelone, Crítica, 1986, p. 14 ; José-Francisco de La Peña, "La política reformista », Historia 16, XIII, n 137, 1987, p. 58 ; Jean Vilar, «Un pessimisme "calculé" : l'introspection économique à Tolède (1616-1628) ", Tolède et l'expansion économique urbaine (colloque de mars 1988), Madrid, Casa de Velázquez, 1991, p. 117-35.

20. Pierre Vilar, «Les primitifs ».

21. Jean Vilar, Literatura, p. 240 et sq.

22. Un résumé dans José-Antonio Álvarez Vázquez, «Arbitristas españoles del siglo XVII ». Il faut y ajouter la publication par l'Instituto de Estudios Fiscales des discours de Pedro Fernández Navarrete, 1982 ; Martin González de Cellorigo et Lope de Deza, 1991.

23. Outre les ouvrages cités en note 12 et les introductions aux éditions critiques des textes d'arbitristes, on pourra consulter les travaux de Miguel-Angel Echevarria Bacigalupe sur Alberto Struzzi, Alberto Struzzi, un precursor barroco del capitalismo liberal, Louvain, Leuven University Press, 1995 ; sur les arbitristes basques installés aux Pays-Bas, ceux d'Henri Méchoulan et José-Antonio Álvarez Vázquez sur Mateo López Bravo, de Luis Miguel Balduque sur Barbón y Castañeda, ou encore les articles consacrés à des arbitristes des colonies américaines publiés par la Revista de Indias depuis deux décennies.

24. Voir les dictionnaires de Maria Moliner, 1966, et de l'Académie Royale.

25. "El problema es ahora hacer la correspondiente distinción y separar el grano de la paja ", José-Luis Abellán, Historia crítica del pensamiento español, volume III : Del Barroco a la Ilustración (siglos XVII y XVIII), Madrid, Espasa-Calpe, 1 ère éd. : 1981, 1988, p. 315.

26. L'association entre arbitrisme et réforme est fréquente : voir à titre d'exemples, Bartolomé Bennassar, "La conscience de la décadence en Espagne. L'appel au roi vers 1600-1620 », Revue d'histoire diplomatique, colloque du centre d'études supérieures de la Renaissance : la croissance de l'Etat moderne (XVe-XVII ${ }^{e}$ siècle), juillet-décembre 1975, p.213-24; Michel Cavillac, introduction à l'Amparo de pobres de Cristóbal Pérez de Herrera, Madrid, Espasa-Calpe, 1975; Benjamín González Alonso, «El Conde-Duque »; Antonio Gutiérrez Escudero, «Acerca del Proyectismo y del Reformismo Borbónico en Santo Domingo », Temas americanistas, nº 13, 1997, p. 17-30 ; JoséFrancisco de la Peña, «La política reformista »; Juan A. Sánchez Belén, La política fiscal en Castilla durante el reinado de Carlos II, Madrid, Siglo XXI, 1996 et «Arbitrismo y reforma monetaria en 
tiempos de Carlos II ", Espacio, tiempo y forma, Serie IV, Historia Moderna, t. V, 1992, p. 135-75; Giovanni Stiffoni, «Tematiche»; Juan-Ignacio Gutiérrez Nieto distingue les arbitristesréformateurs des arbitristes fiscaux, «El pensamiento », p. 235-37.

27. C'est le jugement de Mercedes Barat, pour qui Ángel Manrique, auteur d'un mémoire arbitriste, puede comparecer dignamente junto a ese conjunto de hombres que encuadramos en la mejor tradición arbitrista, es decir, esos pensadores que honestamente quisieron ayudar al reino, y creyeron haber descubierto la solucion de sus males, sin elucubrar sus teorias al calor de un irrefrenable afán de lucro personal, los mismos que, en general [...] no quisieron para si la denominación de arbitristas que una pléyade de embusteros, trepadores o lunáticos habian promovido y divulgado con sus desatinados o interesados proyectos de salvación, «Un texto arbitrista », p. 108, souligné par l'auteur ; MariaLuisa Martinez de Salinas décrit une abundantisima literatura económica, plasmada en obras que, en algunos casos, alcanzan la categoria de auténticos tratados cientificos : ces derniers cohabitent avec les idées les plus fantastiques et irréalisables, "Contribución». Fred Bronner distingue les « arbitrios serios » d'autres « fantasmagoriques », « Tramitación », p. 413.

28. Par exemple pour Luis Valle de la Cerda : "el economista Luis Valle de la Cerda », Eusebio Zarco-Bacas y Cuevas, Relaciones de pueblos del obispado de Cuenca hechas por orden de Felipe II, Cuenca, Biblioteca Diocesana Conquense, 1927, t. I, p. CXxxIX ; « economistas y arbitristas », Josefa Diaz de Diaz Fernández et Fabián Estapé, « La creación de erarios públicos en España : El proyecto de Pedro de Oudegherste. Notas para la historia de la Banca en España. ", Moneda y Crédito, 1956, p. 46 ; « un conocido economista », Valentin Vàzquez de Prada, Historia económica y social de España, vol. III : Los siglos XVI y XVII, Madrid, Confederación General de Cajas de Ahorros, 1978, p. 675-76; « un experto economista », José-Antonio Maravall, Utopía y reformismo en la España de los Austrias, Madrid, Siglo XxI, 1982, p. 115 et 281; Earl J. Hamilton, «La decadencia », p. 131, et Juan-Ignacio Gutiérrez Nieto, «El pensamiento », p. 247, préfèrent aussi le terme economista.

29. José-Luis Abellán, Historia critica, p. 318.

30. Jean Vilar, L'« arbitrista » malgrélui.

31. Cité par José Múñoz Pérez, «Los proyectos sobre España e Indias en el siglo XVIII : El proyectismo como género ", Revista de Estudios Politicos, n 81, 1955, p. 176. Luis Perdices de Blas assume explicitement ce choix de suivre les critères de Pedro Rodriguez de Campomanes : La economia politica de la decadencia de Castilla en el siglo XVII. Investigaciones de los arbitristas sobre la naturaleza y causas de la riqueza de las naciones, Madrid, Editorial Sintesis, 1996, p. 32.

32. Literatura, p. 259.

33. Evaristo Correa Calderón, Registro de arbitristas, p. 19.

34. C'est ce que laissait entendre Fabián Estapé dans un dictionnaire qui fait encore autorité : La literatura económica española se ve dominada hasta bien entrado el siglo XVIII por este tipo de creaciones $y$, en cierto modo, la idea de encontrar la "única solución » a los males que afligen o han afligido al pais, se mantiene constante hasta nuestros tiempos, al extremo de que puede caracterizarse el arbitrismo como una consecuencia del espíritu de la raza. [...] La especial psicologia del arbitrista le fuerza siempre a creer que el auténtico remedio sobrenatural se encuentra cerca [...], « Arbitrismo ».

35. Pour José Múñoz Pérez, le proyectismo du XVIII siècle reflète l'«âme » d'une époque, «Los proyectos ", p. 173.

36. Pour José Múñoz Pérez, les proyectistas se distinguent des arbitristas par leur plus grand professionnalisme. Mieux informés, disposant de données empiriques qu'ils sont en mesure de traiter par la statistique, ils sont aussi capables de prévoir les obstacles qui se présenteront à eux et de prévoir en conséquence une mise en œuvre progressive de la politique prônée. En outre, ils visent le long terme, non le gain facile et rapide, «Los proyectos ». Ces distinctions sont reprises par Pedro Álvarez de Miranda, «Proyectos y proyectistas », María-Luisa Martínez de Salinas «Contribución » et Antonio Gutiérrez Escudero, « Acerca del proyectismo ». Luis Perdices de Blas va plus loin dans l'affirmation d'un hypothétique progrès de la Raison, jugeant le débat des hommes des Lumières « plus élevé » que celui des arbitristes, bien qu'il n'ait choisi d'étudier que 
les meilleurs d'entre eux, La economía política, p. 143 ; Henry Kamen le rejoint sur ce point : Sólo con la eficacia borbónica, el arbitrismo se hizo superfluo. Los datos engañosos y frecuentemente incorrectos presentados por los arbitristas fueron reemplazados por estadísticas elaboradas por funcionarios del régimen borbónico, Vocabulario, p. 15-16.

37. Hoy día sabemos que fueron intelectuales preocupados por los problemas económicos y políticos de España. Aparecen en momentos de crisis y exponen sus opiniones en una serie de avisos, memoriales y expedientes. Proponen soluciones concretas, pero sus ideas no siempre fueron conocidas en su época. Su valor y arrojo sólo comienza a entenderse, Sará Almarza, « Los vocablos ».

38. Juan-Ignacio Gutiérrez Nieto parle de "grupo de pensadores », «El pensamiento », p. 235, $245,250$.

39. C'est en ces termes que Raymond Boudon et François Bourricaud définissent les intellectuels, notion apparue lors de l'affaire Dreyfus, Dictionnaire critique de la sociologie, Paris, PUF, 1982, article « Intellectuels », p. 314-19.

40. Le terme est emprunté à l'expression "donneurs d'avis ». En espagnol, on rencontre des titres tels qu'Avisos ou Advertimientos.

41. José-Luis Abellán, Historia crítica, p. 328.

42. Mais aussi dans un ouvrage de portée générale. Luis Perdices affirme : pretendo mostrar que los autores utilizados para escribir el presente ensayo, realizaron interesantes reflexiones sobre el concepto de riqueza, las causas del atraso sz Espana, el fomento de los sectores productivos, el trabajo productivo y los obstaculos al crecimiento economico, La economia politica, p.20.

43. Jean Vilar revendique le nom de «philologue », L'« arbitrista » malgré lui, p. 37.

44. Anne Dubet, Réformer les finances espagnoles, chap. 6-8.

45. Comme le fait Henri Méchoulan, Mateo López Bravo, un socialista español del siglo XVII, Madrid, Biblioteca de visionarios, 1977. José-Antonio Álvarez Vázquez rappelle à juste titre que la société dont rêve López Bravo reste constituée de corps inégaux, « El arbitrismo de Mateo López Bravo », Hispania, XLIII, 1983, p. 551-79.

46. À l'instar de José-Luis Pérez de Ayala, introduction à Martin González de Cellorigo, Memorial de la politica necesaria y útil restauración a la república de España (1600), Madrid, Instituto de Estudios Fiscales, 1991). Sur ce point : Jean Vilar, L'« arbitrista » malgré lui.

47. C'est ce que fait Luis Perdices: Lo novedoso y paradójico de esos autores es que describen y explican las prácticas más comunes de los comerciantes y banqueros, tales como los préstamos usurarios, sin tomar en cuenta las reglas morales de los teólogos. [...] Son obras que, al igual que la de los arbitristas de los siglos XVI y XVII, tratan de temas económicos sin tener en cuenta las reglas de justicia y equidad de los moralistas escolásticos. [...] se puede decir que los arbitristas castellanos fueron unos autores que realizaron reflexiones sobre la decadencia de Castilla dejando a un lado los problemas morales planteados por los escolásticos, La economia politica, p. 30-31 et 44-45. L'auteur signale pourtant que les arbitristes connaissent les écrits des théologiens (p. 33). J'espère avoir su montrer, dans le cas de Luis Valle de la Cerda, que si un texte d'arbitriste n'est pas un manuel de confesseur, les considérations morales et religieuses ne sont pas pour autant abandonnées lorsque l'on traite de prêt à intérêt, de rentes, ou encore quand on fait de la charité le fondement du lien social, Anne Dubet, op. cit., chap. 8.

48. Earl J. Hamilton, « Spanish banking schemes before 1700 », Journal of Political Economy, $\mathrm{n}^{\circ} 57$, Chicago, 1949.

49. Juan-Ignacio Gutiérrez Nieto, « De la expansión a la decadencia económica de Castilla y León. Manifestaciones. El arbitrismo agrarista.», El pasado histórico de Castilla y León, vol. II : Edad Moderna, Burgos, Junta de Castilla y León, 1983, p. 11-75.

50. Francisco-José Aranda Pérez, «La preocupación « arbitrista » en el seno del ayuntamiento de Toledo por la declinación de la ciudad en un período crítico : 1618-1621 », Toletum, n 29, 1993, p. 201-27. 
51. Felipe Ruiz Martin, «La Banca en España hasta 1782 », El Banco de España, una historia económica, Madrid, 1970, p. 94.

52. Esa literatura económica coetánea constituye el movimiento que se ha llamado arbitrismo, José-Luis Abellán, Historia crítica, p. 313, souligné par l'auteur.

53. Juan-Ignacio Gutiérrez Nieto, « El pensamiento ».

54. Ibid., p. 247.

55. José-Luis Abellán, Historia critica, p. 312.

56. Con el precedente de Luis de Ortiz, Cellorigo da paso a toda una legión de buenos pensadores politicoeconómicos, de arbitristas reformadores, que vuelcan su preocupación sobre la situación del país [...], JuanIgnacio Gutiérrez Nieto, « El pensamiento », p. 250.

57. en González de Cellórigo (sic) [...], encontramos una coherencia formal con el cuantitativismo de la Escuela de Salamanca, y en algunos puntos, hasta una superación del mismo, pero nunca una propuesta de soluciones técnicas a los problemas planteados. Para encontrar algo semejante tenemos que recurrir a los arbitristas propiamente dichos [...]. El primero y más importante de estos autores, a quien podemos considerar como fundador de la Economia política, es Sancho de Moncada, José-Luis Abellán, Historia crítica, p. 317-18, souligné par l'auteur.

58. Juan-Ignacio Gutiérrez Nieto, «El pensamiento ».

59. Juan A. Sánchez Belén, «Arbitrismo y reforma monetaria » et La politica fiscal ; Salvador Albiñana, « Notas sobre decadencia y arbitrismo », Estudis, n² 20, Valence, 1994, p. 9-28.

60. José Múñoz Pérez, « Los proyectos».

61. Sará Almarza, «Los vocablos »; Antonio Dominguez Ortiz, « Nuevos ejemplos de un género muy espanol: el arbitrismo economico del siglo XVII »,Hacienda publica espanola, $\mathrm{n}^{\circ} 100$, 1986,p.275-285 ; Fabien Estapé, «Arbitrismo »; Maria-Luisa de Salinas, «Contribucion »; JoséFrancisco de la Pena, «La politica reformista »

62. L'idée d'une crise généralisée au $\mathrm{XVII}^{\mathrm{e}}$ siècle a bénéficié d'une volumineuse littérature critique au cours des vingt dernières années : Michel Morineau, « Le Siècle », in Pierre Léon (dir.), Histoire économique et sociale du monde, t. II, Les hésitations de la croissance, 1580-1740, Paris, Armand Colin, 1978 ; Ángel García Sanz, «El sector agrario durante el siglo XVII : depresión y reajustes », Historia de España Ramón Menéndez Pidal, Madrid, Espasa-Calpe, t. 23, 1989, p. 161-235.

63. Juan Luis Castellano montre ainsi comment Antolín de la Serna manie l'illusion statistique, «Discurso de Antolín de la Serna ", Chronica Nova, n 17, 1989, p. 349-63.

64. Salvador Albiñana, « Notas sobre decadencia y arbitrismo ».

65. Juan-Ignacio Gutiérrez Nieto, «El pensamiento », p. 235-7, 262. On se reportera aux travaux consacrés aux débuts du duc de Lerme comme favori de Philippe III (1598), à sa chute (1618), à la mise en place de la Grande Junte de Réformation (1622) et à la carrière du comte-duc d'olivares.

66. De même au XVIII ${ }^{\mathrm{e}}$ siècle, les proyectistas sont plus actifs lors des changements de règne, José Múñoz Pérez, «Los proyectos », p. 191.

67. Jean Vilar, L'« arbitrista » malgré lui, p. 23.

68. Juan-Ignacio Gutiérrez Nieto, «El pensamiento », p. 399 et sq. Sur l'« Ecole de Tolède » : Jean Vilar, «Un pessimisme "calculé" »; introduction à la Restauración política de S. de Moncada.

69. Anne Dubet, op. cit., chap. 12. Voir l'index patronymique. Sur Alberto Struzzi on consultera aussi Miguel-Ángel Echevarria Bacigalupe, Alberto Struzzi.

70. «le plus grand arbitriste de son temps » selon John H. Elliott, «El Conde-Duque de Olivares : hombre de Estado ", La España del Conde Duque de Olivares. Encuentro internacional sobre la España del Conde Duque de Olivares celebrado en Toro los días 15-18 de septiembre de 1987, Universidad de Valladolid, 1990, p. 19-30.

71. Comme celle qui en 1608 ordonne la réduction des taux de rente: le raisonnement qui consiste à attribuer l'abandon des activités productives au fait qu'elles rapportent moins que l'oisiveté rentière, en comparant le rendement de la terre à celui de la rente, pourrait avoir été emprunté à Luis Valle de la Cerda ou à Martin González de Cellorigo, BNM, Varios Especiales, 
VE 42-57, Premática para que no se puedan imponer ni fundar juros ni censos al quitar a menos precio de veinte mil maravedis el millar y los de una vida a razón de diez mil maravedís el millar, y por dos vidas a doce.

72. Il fut procurador en 1600 et en 1617-19. En même temps qu'il prenait la parole aux Cortes, il fít circuler des versions manuscrites de son discours, dans lequel il demandait la mise en place de tresoryes inspirées de celles de Luis Valle de la Cerda, AGS, Patronato Real, caja 89, $\mathrm{n}^{\circ} 201$.

73. Anne Dubet, op. cit., p. 272-73.

74. C'est ce que fait de façon catégorique Juan-Ignacio Gutiérrez Nieto.

75. José Múñoz Pérez, « Los proyectos », p. 177-81.

76. Jean Vilar, Literatura, p. 255-57; Beatriz Cárceles de Gea cite des mises en cause de cette illégitimité : « La "justicia distributiva" en el siglo XVII (Aproximación politico-constitucional) », Chronica nova, $\mathrm{n}^{\circ}$ 14, 1984-1985, p. 104-106.

77. J'emprunte la formule énoncée par Jean-Pierre Étienvre lors de ma soutenance de thèse (24/4/98).

78. ACC, vol. XXX, p. 263.

79. Resp. ACC, VI et VII, index et Las rúbricas del Consejo Real y Supremo de las Indias desde la fundación del Consejo en 1524 hasta la terminación del reinado de los Austrías, p. 14 ; Ramón García Mercadal, 1959, t. I, p. 1476.

80. Anne Dubet, op. cit., p. 102, p. 112, p. 155, p. 159, p. 234, p. 238, p. 262, p. 341, p. 347.

81. Fondation Zabálburu (Madrid), caisse $143, \mathrm{n}^{\circ} 216$; Institut Valencia de don Juan (Madrid), envío $45, \mathrm{n}^{\circ} 250$.

82. Jean Vilar, Literatura, p. 178 ; José-Ignacio Fortea Pérez, « Entre dos servicios : la crisis de la hacienda real a fines del siglo XVI. Las alternativas fiscales de una opción política (1590-1601)», Stutia Historica. Historia moderna, $\mathrm{n}^{\circ}$ 17, 1997, p. 63-90.

83. Francisco-José Aranda Pérez, «La preocupación "arbitrista" ».

84. Jean Vilar, « Formes et tendances de l'opposicion sous Olivares : Lisón y Viedma, defensor de la patria ", MCV, $\mathrm{n}^{\circ} 7,1971$, p. 263-94.

85. Jean Vilar, « Formes », p. 276.

86. Françoise Bayard, Le Monde des financiers, $2^{\mathrm{e}}$ partie, chap. 1.

87. Plusieurs avis adressés au Conseil des Indes ont été étudiés dans la Revista de Indias. Sur ceux utilisés par les juntes ou par Olivares, on consultera par exemple John H. Elliott, El Conde-Duque de Olivares, Barcelone, Critica, 1991 (1 ${ }^{\text {ère }}$ éd. 1986) et Juan-Francisco Baltar Rodríguez, Las Juntas. Des références aux avis conservés par le président de Castille Diego de Espinosa à la veille de la suspension des paiements de 1575 chez Esteban Hernández Esteve, Establecimiento de la partida doble en las cuentas centrales de la Real Hacienda de Castilla, Banco de España, $n^{\circ} 14,1986$, p. 146. La fondation Zabálburu et l'Institut Valencia de don Juan (Madrid) conservent nombre d'informations sur les mémoires transmis aux proches de Philippe II et sur l'usage qui en fut fait.

88. Henry Kamen, Vocabulario, p. 14: provenían de una amplia gama de profesiones: soldados, funcionarios, clérigos, comerciantes, juristas; Juan-Ignacio Gutiérrez Nieto, «El pensamiento», p. 241, La sociología de los arbitristas fiscales era variada. Desde eclesiásticos, pasando por abogados y médicos, hasta simples artesanos, la gama era muy extensa. De todas formas, parece ser que el arbitrista, casi por obligación, era el funcionario.

89. Voir l'index patronymique de ma thèse.

90. Françoise Bayard, op. cit., chap. 1.

91. Juan-Ignacio Gutiérrez Nieto fait de l'importance accordée à l'«État» un des signes distinctifs des arbitristes, « El Pensamiento ».

92. Claude Chauchadis, Honneur, morale et société dans l'Espagne de Philippe II, Paris, Éditions du CNRS, 1984 ; Michel Cavillac, Gueux et marchands, chap. IV-3. 
93. Sur ce dernier cas, Anne Dubet, «Guerra económica y financiera entre España y los Países Bajos. El proyecto de erarios del alemán Conrado Rott. ", intervention au XII ${ }^{\circ}$ Congreso de la Asociación de Historia Económica, Session C6, à paraître dans la revue Mayurqa.

94. Sur les factions présentes à la cour de Philippe II, José Martínez Millán, La Corte de Felipe II, Madrid, Alianza Universidad, 1994.

95. Anne Dubet, op. cit., chap. 5-3 et 5-4.

96. Si el arbitrista está en el Buscón y en Teresa de Manzanares, (cuántas novelas picarescas, y de las más importantes no han considerado útil nombrarlo, ni siquiera de pasada) Es esta, sin duda, la prueba más clara de la limitada dimensión del tema, es en el género de la picaresca donde más abundaría si se hubiese convertido como otros tantos en figura indispensable, Jean Vilar, Literatura, p. 148.

97. El arbitrista quedó para siempre incorporado a los tipos literarios característicos de la Edad de Oro : la dueña, el pícaro, la terciadora, José Múñoz Pérez, « Los proyectos », p. 175.

98. L'exemple de Juan Gómez de Bedoya, donné par Juan-Ignacio Gutiérrez Nieto, est significatif, «El pensamiento »; AGS, Consejo y Juntas de Hacienda, leg. 283, 284, 286, 288, 303, 384.

99. Par exemple sous Philippe II la Junte de Réformation étudiée par Alfredo Alvar Ezquerra, « La Junta de Reformación de Felipe II : rezar por el Rey y reorganizar la sociedad (1)», Pablo Fernández Albaladejo (éd.), Monarquia, imperio y pueblos en la España moderna. Actas de la IV Reunión Cientifica de la Asociación Española de Historia Moderna. Alicante, 27-30 de mayo de 1996, Caja de Ahorros del Mediterráneo, Universidad de Alicante, AEHM, 1997, p.641-50 ; ou la Grande Junte (junta grande) de 1591, Anne Dubet, Réformer les finances espagnoles, chap. 10-4.

100. Alfredo Alvar Ezquerra, «Unas "Reglas Generales para remitir memoriales" del siglo XVI ", Cuadernos de Historia Moderna, n 16, 1995, p. 47-71; Bernardo José Garcia Garcia, «El confesor fr Luis Aliaga y la conciencia del rey ", I religiosi a corte. Teologia, politica e diplomazia in Antico Regime. Atti del seminario di studi Georgetown University a Villa «Le Balze». Fiesole, 20 ottobre 1995, Bulzoni Editore, 1995, p. 159-94 ; le projet de «Conseil de la Conscience» de fray Juan de Ribera, en 1602, énonce les règles à suivre dans le traitement des « advertimientos".

101. Francisco-José Aranda Pérez, « La preocupación "arbitrista" ».

102. Le 13 juillet 1626 un certain Juan Juárez propose un réaménagement des fontaines, qu'il se chargerait de mettre en place, réclamant de la ville alguna merced por el beneficio y adbitrio. L'affaire lui est effectivement confiée, à condition que la dépense ne dépasse pas 100 ducats, AVM, AA.CC., 1626, fol. 296-6v.

103. Françoise Bayard, op. cit., $2^{\mathrm{e}}$ partie, chap. 1 .

104. "El pensamiento ", p. 241.

105. Las alteraciones monetarias en España en el siglo XVII, thèse de doctorat dirigée par Alfredo Alvar Ezquerra, 1997.

106. On peut suivre les tractations menées entre Pierre d'Oudegherst, son collaborateur Peter Van Rotis et le roi entre avril et septembre 1576 dans la documentation du Conseil d'État conservée à Simancas (Estado, leg.536, fol. 91; leg.659, fol.103-4; leg.675, fol.28-29, 91 ; leg. 676, fol. 26, 29 ; leg. 677, fol. 28-29, 48, 57, 60).

107. Voir le mémoire des services de Luis Valle de la Cerda, BNM, manuscrit 994. Quand Philippe III décide d'accorder à l'arbitriste l'office de comptable de la Sainte Croisade pour une seconde vie, il reprend les termes de ce mémoire, Archivo Universitario Histórico Provincial de Valladolid, Protocolos, leg. 807, fol. 429v-30v.

108. Le débat porte sur le caractère obligatoire (de justice) ou gracieux de la récompense et sur la mesure avec laquelle le roi doit en user. Antonio M. Hespanha, La gracia del derecho, Madrid, Centro de Estudios Constitucionales, 1993 ; John H. Elliott et José-Francisco de la Peña, Memoriales y cartas del Conde-Duque de Olivares, 2 t., Madrid, Ediciones Alfaguara, p. 139-41.

109. y siendo de cosas nuevas que vengan a mi noticia por su industria y [sic] invención, y de que no se haya usado hasta agora ni se tenga razón en nuestros libros, AGS, Estado, leg. 677, fol. 48. 
110. Lo bien que vuestra merced trabaja y que bien lo tengo ententido y no era menester ver nuevos papeles y pruebas para conocer la gracia que tiene en el descifrar, y como se ofrezcan ocasiones no dejaré de hacer en ellas por mi parte todo (?) buen oficio conforme a nuestra amistad y a la buena opinión en que tengo a vuestra merced, don Juan de Idiáquez à Luis Valle de la Cerda, San Lorenzo del Escorial, 27/08/1586, AGS, Estado, leg. K 1448.

111. pues cada dia me hallo con más trabajos y obligaciones, pero tengo tantas a Vuestra Señoria para en esta ocasión concluir con Su Majestad lo del titulo de secretario que tantos años ha que deseo, Luis Valle de la Cerda à don Juan de Idiáquez, 03/12/1595, BNM, Ms 994, fol. 98.

112. Josefa Diaz de Díaz Fernández et Fabián Estapé, «La creación de Erarios públicos ».

113. Par exemple John H. Elliott ou Juan A. Sánchez Belén. Voir aussi José-Ignacio Fortea Pérez, «Entre dos servicios ».

114. Françoise Bayard, op. cit., $2^{\mathrm{e}}$ partie, chap. 1 .

115. Pour une interprétation des contrats des millones et des relations qui se nouent entre la Couronne, les villes et les Cortes au XVII ${ }^{\mathrm{e}}$ siècle : Charles Jago, « Fiscalidad y cambio constitucional en Castilla », Carmen María Cremades Griñán et José-Ignacio Fortea Pérez (dir.), Politica y hacienda en el Antiguo Régimen. II réunión cientifica. Asociación española de Historia Moderna (1992), vol. I, Murcie, Universidad de Murcia, 1993, p. 117-32 ; José-Ignacio Fortea Pérez, « Reino y Cortes. El servicio de millones y la reestructuración del espacio fiscal en la Corona de Castilla (1601-1621) », Op. cit., p. 53-82 ; Irving A. A. Thompson, «Cortes y ciudades: tipología de los procuradores (extracción social, representatividad ", Las Cortes de Castilla y León en la Edad Moderna. Actas de la Segunda Etapa del Congreso Científico sobre la Historia de las Cortes de Castilla y León. Salamanca, del 7 al 10 de Abril de 1987, Cortes de Castilla y León, 1989, p. 191-248; Pablo Fernández Albaladejo, Fragmentos de Monarquía, Madrid, Alianza Universidad, 1993.

116. Anne Dubet, op. cit., chap. 11-2.

117. Carmen Sanz Ayán, Los banqueros de Carlos II, Valladolid, Universidad de Valladolid, 1989.

118. Andrée Chauleur, «Le rôle des traitants "; Daniel Dessert, "Finances et société au XVII ${ }^{\mathrm{e}}$ siècle : à propos de la Chambre de Justice de 1661 ", Annales ESC, nº 4, 1974, p. 847-71.

119. Jean Vilar, Literatura, p. 62, 89, 153, 167-68, 186.

120. Charles Jago, «The Influence of Debt on the Relations Between Crown and Aristocracy dans Seventeenth-Century Castile », Economic History Review, XXVI, 1973, p. 218-36 ; Juan A. Sánchez Belén, La politica fiscal, p. 99-114.

121. Ainsi, Pierre d'Oudegherst puis Luis Valle de la Cerda modifient constamment leur description des tresoryes entre 1576 et 1600. En 1622, Manuel López Pereira, partisan du projet de la Grande Junte de Réformation, propose un aménagement substantiel : les monts-de-piété seraient tenus par des financiers privés. En 1625, plusieurs arbitristes proches du comte-duc imaginent des versions modifiées des tresoryes, Anne Dubet, op. cit.

122. Sur l'opposition wébérienne entre administration traditionnelle et administration moderne : Julien Freund, Sociologie de Max Weber, Paris, PUF, 1966, p. 190-214.

123. Pour une synthèse des dernières controverses historiographiques sur la notion d'État moderne : Jean-Frédéric Schaub, «L'histoire politique sans l'État : mutations et reformulations ", Historia a debate, t. III, 1995, p. 217-35; Jésus Vallejo, "Paratonia de la historia juridica », MCV, XXXI-2, Madrid, 1995, p. 109-41. 